\title{
Guidelines for the Psychosocial and Bereavement Support of Family Caregivers of Palliative Care Patients
}

\author{
Peter Hudson, R.N., B.N. (Hons), Ph.D.,,2 Cheryl Remedios, B.A. (Hons), DPsych (Health), \\ Rachel Zordan, B.Sc. (Psych), GDipSc (Psych), Ph.D., \\ Kristina Thomas, BPsych (Health), Doctor of Psychology (Health), \\ Di Clifton, M.B.B.S., BMedSci (Hons), M.P.M., FRANZCP, ${ }^{3}$ Michael Crewdson, B.A., BBehSci (Hons Psych), M.Sc., ${ }^{4}$ \\ Christopher Hall, M.A., GDipAdChPsych, B.Ed., ${ }^{5}$ Tom Trauer, B.A. (Hons), Ph.D., ${ }^{3,4,6}$ Amanda Bolleter, B.A., B.S.W., \\ David M. Clarke, MBBS, M.P.M., Ph.D., ${ }^{6}$ and Catherine Bauld, B.A. (Psych), GDipAdolPsych, Ph.D.1
}

\begin{abstract}
Background: Support for family caregivers, including bereavement follow-up, is a core function of palliative care. Many caregivers acknowledge positive aspects associated with the role; however a considerable proportion will experience poor psychological, social, financial, spiritual, and physical well-being and some will suffer from complicated grief. Many family caregivers have unmet needs and would like more information, preparation, and support to assist them in the caregiving role. There is a shortage of evidence-based strategies to guide health professionals in providing optimal support while the caregiver is providing care and after the patient's death. Purpose: To develop clinical practice guidelines for the psychosocial and bereavement support of family caregivers of palliative care patients.

Methods: (1) Literature review; (2) focus groups and structured interviews with key stakeholders within Australia; (3) national and international expert opinion to further develop and refine the guidelines using a modified Delphi process; and (4) endorsement of the guidelines from key palliative care, caregiver, and bereavement organizations (national and international).

Results: The guidelines were developed for multidisciplinary health care professionals and clinical services commonly involved in caring for adult patients receiving palliative care in a variety of care sites throughout Australia. These consensus-based guidelines have been endorsed key Australian and international organizations. Conclusions: The guidelines may prove valuable for the international palliative care community and for generalist health care providers who occasionally care for palliative care patients. Research is recommended to explore the uptake, implementation, and effectiveness of the guidelines.
\end{abstract}

\section{Introduction}

$\mathbf{T}$ He World Health Organization advocates that palliative care should not only improve the quality of life for patients but also for their families. ${ }^{1}$ Comprehensive support for bereaved family members is also expected. ${ }^{1}$ Family caregivers within the context of palliative care have been defined as any relative, friend, or partner who has a significant relationship and provides assistance (physical, social, and/or psychological) to a person with a life-threatening, incurable illness. $^{2}$

In many countries palliative care practice standards advocate that family caregivers' needs be assessed and responded to with relevant psychosocial supports. ${ }^{3,4}$ Ensuring that family caregivers' needs are appropriately assessed is one of the top ten quality markers for end-of-life care. ${ }^{5}$

There are many reasons why family caregivers should be offered psychosocial support. ${ }^{6}$ Caregivers typically are prone

\footnotetext{
${ }^{1}$ Centre for Palliative Care at St. Vincent's Hospital \& a collaborative centre of The University of Melbourne, Melbourne, Australia.

${ }^{2}$ Queen's University, Belfast, United Kingdom.

${ }^{3}$ St. Vincent's Hospital, Melbourne, Australia.

${ }^{4}$ The University of Melbourne, Melbourne, Australia.

${ }^{5}$ Australian Centre for Grief and Bereavement, Melbourne, Australia.

${ }^{6}$ Monash University, Melbourne, Australia.

${ }^{7}$ Department of Health, Victoria, Australia.

Accepted February 1, 2012.
} 
to physical and psychological morbidity; ${ }^{7-12}$ are responsible for numerous tasks, such as symptom management; ${ }^{8}$ are financially disadvantaged; ${ }^{8,9,13}$ become socially isolated $;{ }^{14}$ report unmet needs; ${ }^{15}$ experience psychological distress at similar levels to patients; ${ }^{16}$ are often not aware there is support available to them; ${ }^{4}$ have needs equal to and/or greater than the needs of patients; ${ }^{17}$ have very limited prior exposure to death and dying;, ${ }^{8,15}$ are often excluded from information and care planning and consequently feel underprepared for their role ${ }^{15,18}$ can improve the care of palliative care patients; ${ }^{19-21}$ have the potential (with suitable support) to gain positive outcomes from the role, , 15,22 are pivotal to achieving 'successful' home care (where most people prefer to die); ${ }^{21,23,24}$ and make a very significant economic contribution to health care. ${ }^{25}$

Even though there is a requirement for health and psychosocial care professionals to support family caregivers, the reality is that in many instances this support is suboptimal. Approximately $50 \%$ of caregivers are below population norms on physical health, and rates of depression and anxiety (based on screening measures) in up to $44 \%$ of caregivers have been reported. ${ }^{7-12}$ Complicated grief where debilitating grief is experienced for an extended period is noted in approximately $10 \%$ to $20 \%$ of family caregivers. ${ }^{26,27}$ A lack of preparedness for the patient's death has been associated with complicated grief. ${ }^{28}$

Unmet needs are consistently cited in the literature that relate to the lack of psychological support for family caregivers. ${ }^{29}$ Emotionally burdened family members can hinder patients' adjustment to their illness. ${ }^{30}$ Clinicians may normalize the distress or lack the awareness and skill to differentiate distress from sadness, contributing to under-detection; limited management options may also discourage detection..$^{31}$ In a review of interventions to reduce caregiver strain and burden in those caring for cancer patients no suitable intervention could be identified as recommended practice. ${ }^{32}$ Recent systematic reviews of interventions for family caregivers reveal that empirical inquiry regarding effective ways to provide psychosocial support to family caregivers is still in its infancy. 33,34

There is evidence that implementing clinical guidelines, protocols, and tools facilitates improvements in care. Examples include timely referrals to palliative care, reduced emergency department admissions, decreased length of stay in hospital, and an increase in the proportion of deaths at home or in a non-acute care facility. ${ }^{35-38}$

Given this background of less than optimal support for family caregivers and limited interventions to assist this population, we aimed to develop guidelines for the psychosocial and bereavement support of family caregivers of palliative care patients. The primary target audience for the guidelines were multidisciplinary health care professionals commonly involved in caring for adult patients receiving specialist palliative care (home, hospital, hospice) throughout Australia. It was also intended that the guidelines be designed to have relevance for generalist health care providers who occasionally provide palliative care and for the international palliative care community.

\section{Guideline Development Process}

Development of the guidelines entailed: (1) literature review; (2) draft guidelines prepared by the project team based on literature review; (3) focus groups and structured inter- views with key stakeholders within Australia; (4) revision of guidelines based on findings from focus groups and interviews; (5) national and international expert opinion to further develop and refine the guidelines using a modified Delphi process; (6) revision of guidelines based on findings from the Delphi process; and (7) obtaining endorsement of guidelines by key organizations (national and international).

\section{Literature review}

The literature review was carried out to evaluate information published in the last decade related to family caregivers of palliative care patients. The objectives were (1) to outline the experiences, impact, and needs of family caregivers during care provision for their relative and into bereavement; (2) to review evidence-based psychosocial and bereavement support interventions for family caregivers of palliative care patients; and (3) to use the review as a reference for evaluating the levels of evidence for the guideline development process. The specific search strategy, inclusion criteria, and results are outlined elsewhere. ${ }^{33,39}$

\section{Development of first draft of guidelines and review of levels of evidence}

A meeting with the project investigators was arranged to review and further develop the first draft of the clinical guidelines. The project team consisted of a multidisciplinary group of academics, clinicians, a policy maker, and manager who had significant expertise in palliative and/or bereavement care. Prior to the meeting, project investigators were sent a copy of the literature review and a draft of the guidelines for review.

The evidence to support the guidelines was rated in accordance with the National Health and Medical Research Council (NHMRC) (Australia). ${ }^{40}$ The NHMRC sets out the basis for rating key components of the body of evidence for each recommendation (guideline). Due to the paucity of highlevel evidence in this field, and the subsequent need to draw on evidence outside of the gold-standard randomized controlled trial, two further levels of evidence were added. Qualitative studies were acknowledged, and expert opinion was included to support some of the guidelines. The process incorporated one reviewer who rated literature based on the above mentioned criteria. Rated literature was then randomly selected $(50 \%)$ for further review by two of the authors who were blinded to the level of evidence rating. Any discrepancies were discussed amongst the group until consensus was achieved.

\section{Focus group and interviews}

Ethics approval to conduct this phase of the project was gained from St Vincent's Hospital (Melbourne). A panel of state and national key stakeholders from Australia were identified by the project team (via purposive sampling) and invited by letter to participate in either a focus group or structured interview that was designed to obtain opinion and feedback on the second draft of the guidelines. Stakeholders included representatives from multiple health care disciplines currently involved in palliative or bereavement care, policy makers, and consumers. Five stakeholders participated in a focus group, nine participated in an interview, and two 
provided written feedback. Focus groups participants included a clinical psychologist, pastoral care worker, a palliative care nurse with specialisation in aged care, a multicultural health representative and a palliative care volunteer. The focus group was audio taped for the purpose of research analysis. Individual interviews were conducted with a palliative care physician, oncology social workers $(n=2)$, caregiver support advisor (Carers' Australia), psychiatrist, palliative care nurse practitioner, palliative care service manager, psychooncology service manager, and support service manager (Cancer Council New South Wales (NSW). Further written feedback was provided by a consumer representative and a support service manager (Cancer Council NSW).

The majority of respondents agreed that the guidelines would be helpful to patients, families, and professionals working in clinical palliative care. The points exemplifying the major findings from the focus group and interviews are outlined in Table 1.

\section{Revision of guidelines following focus group and interviews}

A summary of the findings from the focus group and interviews were presented to the project investigators. The investigators and project officers discussed the findings and further revised the guidelines based on the comments and recommendations made, and a third draft of the guidelines was developed. This version included a set of principles to underpin the guidelines.

\section{Modified Delphi process}

A modified Delphi process was conducted to obtain consensus on the guidelines from national and international experts in the field of palliative care. Ethics approval was provided by St Vincent's Hospital (Melbourne). Invitations to participate in the process were sent to national and international experts $(n=82)$ in the fields of palliative and bereavement care. Participants were selected by members of the

Table 1. Summary of Recommendations FROM FOCUS GROUP AND INTERVIEWS

- The majority of respondents wanted more detailed clinical practice examples based on various situations and circumstances, for example, culturally and linguistically diverse populations and noncancer illnesses.

- Some participants felt the guidelines were an ideal model of practice and wanted to know how the guidelines aligned with other frameworks and national standards.

- There were requests for further information on assessment practices including examples of screening tools.

- Some respondents stated there was too much focus on complicated grief or prolonged grief disorder. The recognition of other mental health issues was considered important.

- The importance of linking in with other care agencies and community services beyond specialist palliative care was highlighted.

- Some questioned whether it was realistic to conduct the bereavement follow-up suggested in the guidelines in all palliative care settings.

- Other general comments made by respondents related to terminology and expression. project team on the basis that they were recognized as an authority or expert in palliative and/or bereavement care.

The guidelines and a set of principles to underpin them developed by the research team were accompanied by a questionnaire comprising four parts. Part A was designed to collect participant information including gender; country; region (metropolitan, regional city, or rural area); current occupation; primary profession; and main area of speciality of participants. Part B consisted of one qualitative question inviting participants to review the principles $(n=13)$ and comment on the clarity and comprehensiveness of the principles. Part C invited participants to rate their level of agreement with each guideline and to provide feedback on each guideline. Part $C$ questions were answered using a Likert scale of 1-5 ( 1 = strongly disagree and $5=$ strongly agree), with a sixth answer option of "don't know." Four qualitative questions were included to ascertain further feedback from participants on each guideline and to encourage participants to provide further examples of good clinical practice and the source from which the example was generated (e.g., literature or personal experience). Part D provided participants with the opportunity to add general comments regarding the guidelines. The Delphi process was administered via the Internet using LimeSurvey ${ }^{\circledR}$ software.

There is no standard level of consensus that should be attained by an expert panel involved in a Delphi process. ${ }^{41}$ The research group made an a priori decision that a consensus required $75 \%$ or more of the members of the expert group to agree or strongly agree with the proposed guideline. Participant responses were evaluated and summarized to identify those guidelines with a high level of agreement and those for which there was no agreement or clear disagreement. To determine support or otherwise for each guideline, scores for each question were totalled, with a higher score indicating a more positive response to the guideline.

Demographic data were subject to descriptive analysis (frequencies and percentages). Data were analyzed for each guideline separately (frequency, medians, means, standard deviations) to enable decisions to be made as to whether consensus had or had not been reached. Written comments were thematically analysed and data circulated to the research group for consideration. When consensus had been reached (greater or equal to $75 \%$ in agreement), the guideline was considered to be completed.

Fifty-two experts registered to participate in the survey (recruitment rate $(52 / 82=63 \%)$ and 26 completed the survey, corresponding to a response rate of $50 \%$. The majority of participants were female $(n=19,73 \%)$ and worked in a major metropolitan area $(n=20,77 \%)$. Many participants were international $(n=16,62 \%)$, with 10 participants based in Australia (38\%). Generally, participants were academics $(n=20$, $77 \%)$, with five $(19 \%)$ in clinical roles, and one participant in clinical management. Palliative care was the most common specialization $(n=17,65 \%)$, followed by bereavement $(n=5$, $18 \%$ ), oncology, aged care, and psychooncology (all $n=1,4 \%$ ).

Feedback on the principles was very positive with most participants $(n=24)$ agreeing with them and providing only minor feedback in terms of phraseology modifications. Five participants commented that the use of "primary family caregiver" could be exclusionary to other family members and caregivers/support persons who may not be recognized as family; alternate terms were suggested. 
Analysis of guideline rating data revealed consensus was reached for all 20 guidelines, with more than $75 \%(M=87 \%)$ of the 26 expert panel members rating each guideline positively.

Overall, participants agreed that the guidelines were important, practical, realistic, relevant, and applicable to both specialist palliative care and general health care providers. Qualitative feedback provided generally focused on the details associated with the guidelines. For example, respondents agreed with Guideline 10, Determine the current state of and risk for poor psychological health and/or prolonged grief and plan relevant intervention(s), with a consensus level of $92.3 \%$. However, many respondents stated that the screening tools utilized should be reliable, valid, and administered by appropriately trained staff. All qualitative feedback was reviewed by the research team and relevant alterations were made. As consensus was reached in the first round of the Delphi process, a second review round by the expert panel was not required.

\section{Endorsement of the guidelines}

Official endorsement of the guidelines was provided by Beyondblue, the national depression initiative; Caregivers Australia; Caregivers Victoria; Palliative Care Australia; Palliative Care Victoria; Australian Centre for Grief and Bereavement; St Christopher's Hospice, London (United Kingdom); and the International Observatory of End of Life Care (United Kingdom).

\section{The Guidelines}

The final version of the guidelines incorporated background/rationale, purpose, principles, glossary, levels of evidence system, recommended key reading, and reference list. The principles underpinning the guidelines are outlined in Table 2. Twenty guidelines (see Table 3) were included and set up in the following way: guideline statement; aim of guideline; highest grade of evidence; key supportive references (linked to national palliative care standards), and an example of good practice. A summary of the guideline statements is in Table 3. A copy of the complete guidelines, which includes the methodology and the literature review that supports the guidelines, is available via www.centreforpallcare.org.

\section{Dissemination}

To foster uptake of the guidelines, the following dissemination strategies were employed: (1) hardcopy sent to all palliative care services in Australia; (2) email sent to all services and palliative care peak bodies (organizations that promote and/or advocate improvements in palliative care) in Australia and to national and international experts advising them that the guidelines are available electronically for free download at www.centreforpallcare.org; (3) launch by Victorian Minister for Health (Victoria, Australia in April 2011); (4) presentation at the Palliative Care Australia Congress (Cairns, September 2011); (5) endorsing institutions promoting the guidelines via their networks; and (6) availability on

Table 2. Principles for Family Caregiver Support

1. The crucial role of family caregivers ${ }^{1}$ in the care of the patient is acknowledged by the interdisciplinary team, and accordingly, family caregiver and bereavement support should be fully incorporated into service philosophy and standards of care.

2. Family caregivers should be offered needs assessments and access to relevant psychosocial support.

3. The focus of support should be the primary family caregiver(s), as identified by the patient. Where additional resources and skills are readily available, support is extended to other family caregivers.

4. Family caregiver and bereavement support is provided using resources based on the available evidence and best practice guidelines.

5. The focus of advice and support provided to the family caregiver(s) should be based on caregivers' choice and emphasize strategies to optimize their own well-being.

6. Communication with family caregiver(s) should be conducted in a manner that provides direct and clear information in a language they understand via a combination of mediums including verbal, written, or multimedia. Relevant confidentiality and legal requirements should be considered.

7. It is recommended that discussions around preparedness for the patient's death are responsive to the individual needs of the family caregiver(s). This may require discussing issues in stages according to the patient's declining physical condition.

8. Health care professionals and volunteers working with family caregivers should receive appropriate and ongoing training, support, and supervision relevant to their level of involvement. Training should be based on national standards and evidence-based guidelines.

9. Health care professionals, patients, and family caregivers should be aware of the limits of palliative and bereavement services. Referral to external specialist services or individuals should be undertaken when appropriate.

10. Family caregiver and bereavement support is provided with respect to individuals and their needs within a safe, confidential, and ethical approach that is sensitive to the caregivers' experience, culture, and social environment.

11. Although poor bereavement outcomes are associated with identified risk factors, there is minimal evidence to support routine intervention with all bereaved people.

12. Comprehensive care planning by the interdisciplinary team, involving the patient and family caregiver(s), is an ongoing process, rather than a single event.

13. All relevant discussions, assessments, and outcomes related to the patient and family caregiver(s) should be clearly documented in the most appropriate location (as per the service protocol).

14. A clear process for consumer complaints and feedback with regard to the quality of health professional support should be easily accessible to patients and family caregivers.

\footnotetext{
${ }^{1}$ These principles and guidelines focus on the relative or friend who predominantly supports the patient - the primary family caregiver. In these guidelines the term "primary family caregiver" equates to family caregiver or family caregivers.
} 
Table 3. Summary of Guideline Statements

Part 1: Setting Up Family Caregiver Support

Guideline 1

Guideline 2

Guideline 3

Guideline 4

Guideline 5

Guideline 6

Guideline 7

Guideline 8

Guideline 9

Guideline 10

Guideline 11

Guideline 12

Guideline 13

Guideline 14

Guideline 15

Guideline 16

Guideline 17

Guideline 18

Guideline 19

Guideline 20
Once a patient has agreed to receive palliative care, inform the patient that the role of palliative care is also to support family caregiver(s). The family caregiver(s) will be a person(s) who the patient perceives to be his or her most important support person(s). This may be a family member, partner, or friend and may not necessarily be his or her next of kin.

Ask the patient to identify the primary family caregiver. Where only one family caregiver is identified, ask the patient if there is another family member or friend who may be willing to be approached and act as an additional family caregiver. Discuss the patient's preferences for the involvement of the family caregiver in medical and care planning discussions and note this in the medical record and care plan.

Confirm with the family caregiver(s) that they understand the patient has nominated them for this role. Explain the typical role and responsibilities of the family caregiver and confirm they are willing to accept this responsibility and note this in the medical record. Discuss any concerns that the family caregiver may have in accepting this role (including possible conflicts with other family members).

Discuss advance care planning with the patient and family covering any implications relating to the legal responsibilities of the family caregiver(s).

Recognize the family caregiver as an important source of information about the patient. Gather information from the family caregiver about his or her experience as a support person for the patient, including any information (where pertinent) regarding the patient believed possibly to be important for the health care professional team to know about.

Explain to the family caregiver(s) what services and resources can be provided by the palliative care service so that realistic expectations are established.

\section{Part 2: Assessing Need and Establishing a Plan of Care}

Whenever possible, convene a family meeting/case conference, including the patient if practicable.

Conduct a needs assessment with the family caregiver(s). This should include psychological and physical health, social, spiritual, cultural, financial, and practical elements.

Once the family caregiver(s)' needs are assessed, develop a plan of action with involvement from the family caregiver(s). Initiate the appropriate interventions as pertinent.

Based on discussion with the family caregiver(s), determine the current state of and risk for poor psychological health and/or prolonged grief, and plan relevant intervention(s).

\section{Part 3: Preparing for Death}

When appropriate, assist the family caregiver(s) in how to recognize signs that death may be imminent and the potential implications for the patient's care requirements.

When death appears imminent ensure the family caregiver(s) are aware and assess preparedness for death.

Confirm with the family caregiver(s) the type of support they may desire in the lead-up to death (e.g., last hours, days) and/or immediately after.

The interdisciplinary team identifies a means of communicating with the family caregiver(s) to determine short-term and long-term post-death responses. Potential external bereavement support services are identified if required.

\section{Part 4: Bereavement Support}

Relevant members of the interdisciplinary team are advised of the patient's death in a timely fashion.

As soon as practicable after the patient's death, a member of the interdisciplinary team should contact the family caregiver(s) to offer condolences and respond to queries. If death occurred at home, assess the need for a home visit.

Develop a preliminary bereavement care plan based on the needs of the family caregiver(s), the predeath risk assessment, and the circumstances of the death (e.g., unexpected or traumatic).

Contact the family caregiver(s) and other family members (as appropriate) to assess needs at three to six weeks post-death and adapt bereavement care plan accordingly.

Conduct a follow-up assessment of the family caregiver(s) and other family members (if appropriate) six months post-death.

Following the death of a patient, the interdisciplinary team should have a discussion (at an appropriate time) about the quality of care provided to the patient and family caregiver(s) and the nature of the death.
Australia's web-based knowledge transfer initiative, at www.caresearch.com.au.

\section{Discussion and Conclusion}

The Guidelines for the Psychosocial and Bereavement Support of Family Caregivers of Palliative Care Patients were developed in recognition that support for family caregivers is commonly less than satisfactory. The guideline development process was comprehensive and drawn from the latest evidence, input from multidisciplinary care providers, consumers, policy makers, and consensus-based expert opinion both nationally and internationally and linked to relevant practice standards. A variety of cultural groups are represented in the examples of best practice written to support the guidelines. Effort was also taken to include examples of best practice addressing nonmalignant disease and death occurring outside of a specialist palliative care setting. 
There are several limitations associated with the guidelines. First and foremost, there is currently no formal requirement for services to use the guidelines. Second, the response and completion rates associated with the Delphi process were less than optimal. The length of the survey may have been an impediment to completion. Third, a lack of high-level evidence in this field meant alternate evidence, including qualitative literature and expert opinion, was occasionally required to support the guidelines. Fourth, we acknowledge that it may not be possible for all health care professionals to practice in accordance with all the guidelines. Nonetheless, we advocate that where possible the guidelines should be followed and an effort should be made (e.g., seeking additional resources where relevant) to meet the guidelines in the future. Finally, although the guidelines have been disseminated widely thus far, there is no funded implementation or evaluation plan; this is strongly advocated. In particular it would be helpful to explore strategies for the uptake and impact of the guidelines for generalist health care providers and the international palliative care community. We encourage people to adapt the guidelines (with due acknowledgment) to meet specific local needs as pertinent and to examine their impact.

These limitations reinforce the need for subsequent research and evaluation. For example, conducting a bereavement risk assessment using appropriate and reliable methods and then offering effective bereavement interventions requires further investigation. It is intended that these guidelines will improve the quality of support provided to family caregivers of people receiving palliative care. It would be ideal if policy makers and service managers promoted their use. Additional research is required to determine if this goal can be achieved and to determine the impact of the guidelines.

\section{Acknowledgments}

Funding source: Beyondblue: the national depression initiative

Research assistant: Dawn Whithall

Organizations that endorsed the guidelines: Beyondblue: the national depression initiative; Carers Australia; Carers Victoria; Palliative Care Australia; Palliative Care Victoria; Australian Centre for Grief and Bereavement; St Christopher's Hospice; London International Observatory of End of Life Care (United Kingdom); Participants who were involved in focus groups, interviews, and Delphi survey.

\section{Author Disclosure Statement}

No competing financial interests exist.

\section{References}

1. World Health Organisation. National cancer control programmes: Policies and managerial guidelines, 2nd ed. Geneva: WHO, 2002.

2. Hudson P, Payne S: The future of family caregiving: Research, social policy and clinical practice. In: Hudson P, Payne S (eds). Family Carers in Palliative Care: A guide for health and social care professionals. Oxford: Oxford University Press, 2009:277-303.

3. National Institute for Clinical Excellence: Guidance on cancer services: Improving supportive and palliative care for adults with cancer. The manual. London: National Institute for Clinical Excellence, 2004.

4. Help the Hospices: Identifying carers' needs in the palliative setting. Help the Hospices, 2009.

5. UK Department of Health: End of Life Care Strategy: Quality Markers and Measures for End of Life Care. London: Department of Health, 2009:52.

6. Hudson P, Payne S: Family caregivers and palliative care: Current status and agenda for the future. J Palliat Med 2011.

7. Grov EK, Dahl AA, Moum T, Fossa SD: Anxiety, depression, and quality of life in caregivers of patients with cancer in late palliative phase. Ann Oncol 2005;16(7):1185-1191.

8. Girgis A, Johnson C, Currow D, Bowman D, Waller A, Kristjanson LJ, Mitchell G, Yates P, Neil A, Kelly B, Tattersall M: Palliative Care Needs Assessment Guidelines. Newcastle, NSW: The Centre for Health Research \& Psycho-oncology, 2006.

9. Mockford C, Jenkinson C, Fitzpatrick R: A Review: Carers, MND and service provision. Amyotroph Lateral Scler 2006;7(3):132-141.

10. Low JTS, Payne S, Roderick P: The impact of stroke on informal carers: A literature review. Soc Sci Med 1999;49(6): 711-725.

11. Hudson PL, Thomas K, Trauer T, Remedios C, Clarke D: Psychological and social profile of family caregivers on commencement of palliative care. J Pain Symptom Manage 2011;41(3):522-534.

12. Dempster M, McCorry NK, Brennan E, Donnelly M, Murray LJ, Johnston BT: Psychological distress among family carers of oesophageal cancer survivors: The role of illness cognitions and coping. Psychooncology 2010.

13. McLaughlin D, Sullivan K, Hasson F: Hospice at home service: The carer's perspective. Support Care Cancer 2007; 15(2):163-170.

14. Stoltz P, Uden G, Willman A: Support for family carers who care for an elderly person at home: A systematic literature review. Scand J Caring Sci 2004;18(2):111-119.

15. Andershed B: Relatives in end-of-life care, Part 1. A systematic review of the literature [of] the five last years, January 1999-February 2004. J Clin. Nurs 2006;15(9):1158-1169.

16. Zwahlen D, Hagenbuch N, Carley MI, Recklitis CJ, Buchni S: Screening cancer patients' families with the distress thermometer (DT): A validation study. Psychooncology 2008; 17(10):959-966.

17. Monroe B, Olivere D: Communicating with Family Carers. In: Hudson P, Payne S (eds). Family Carers in Palliative Care: A Guide for Health and Social Care Professionals. New York: Oxford University Press, 2009:1-20.

18. Hebert RS, Schulz R, Copeland VC, Arnold RM: Preparing family caregivers for death and bereavement. Insights from caregivers of terminally ill patients. J Pain Symptom Manage 2009;31(1):3-12.

19. Solomon MZ: The wisdom and necessity of focusing on family. J Palliat Med 2008;11(3):408-409.

20. Mularski RA, Rosefeld K, Coons SJ, Dueck A, Cella D, Feuer DJ, Lipscomb J, Karpeh MS Jr, Mosich T, Sloan JA, Krouse RS. Measuring outcomes in randomized prospective trials in palliative care. J Pain Symptom Manage 2007;34(1S):S7-S19.

21. Demiris G, Oliver DRP, Courtney KL, Porock D: Use of technology as a support mechanism for caregivers of hospice patients. J Palliat Care 2005;21(4):303-309.

22. Hudson P: Positive aspects and challenges associated with caring for a dying relative at home. Int J Palliat Nurs 2004;10(2):58-66. 
23. Docherty A, Owens A, Asadi-Lari M, Petchey R, Wiliams J, Carter YH: Knowledge and information needs of informal caregivers in palliative care: A qualitative systematic review. Palliat Med 2008;22(2):153-171.

24. Gomes B, Higginson I: Factors influencing death at home in terminally ill patients with cancer: Systematic review. BM] 2006;332(7540):515-521.

25. Grande G, Stajduhar K, Aoun S, Toye C, Funk L, AddingtonHall J, Payne S, Todd C: Supporting lay carers in end of life care: Current gaps and future priorities. Palliat Med 2009; 23:339-344.

26. Prigerson HG, Bierhals AJ, Kasl SV, Reynolds CF, 3rd, Shear MK, Newsom JT, Jacobs S: Complicated grief as a disorder distinct from bereavement-related depression and anxiety: A replication study. Am J Psychiatry 1996;153(11):1484-1486.

27. Stroebe M, Hansson $R$, Schut $H$, Stroebe W (eds). Handbook of Bereavement Research and Practice: Advances in Theory and Intervention. Washington, DC: American Psychological Association, 2008.

28. Barry LC, Kasl SV, Prigerson HG: Psychiatric disorders among bereaved persons: The role of perceived circumstances of death and preparedness for death. Am J Geriatr Psychiatry 2002;10(4):447-457.

29. Harding R: Carers: Current research and developments. In: Firth P, Luff G, Oliviere D (eds). Facing Death: Loss, Change and Bereavement in Palliative Care, 1st ed. Maidenhead, Berkshire: Open University Press, 2005:150-166.

30. Zwahlen D, Hagenbuch N, Jenewein J, Carley MI, Buchi S: Adopting a family approach to theory and practice: Measuring distress in cancer patient-partner dyads with the distress thermometer. Psychooncology. 2010.

31. Thekkumpurath P, Venkateswaran C, Kumar M, Bennett MI: Screening for psychological distress in palliative care: A systematic review. J Pain Symptom Manage 2008;36(5):520-528.

32. Honea NJ, Britnall R, Given B, Sherwood P, Colao DB, Somers SC, Northouse LL. Putting evidence into practice: Nursing assessment and interventions to reduce family caregiver strain and burden. Clin J Oncol Nurs 2008;12(3):507-516.

33. Hudson P, Remedios C, Thomas K: A systematic review of psychosocial interventions for family carers of palliative care patients. BMC Palliat Care 2010;9(1):17.
34. Candy B, Jones L, Drake R, Leurent B, King M: Interventions for supporting informal caregivers of patients in the terminal phase of a disease. Cochrane Database Syst Rev 2011(6).

35. Dudgeon DJ, Knott C, Chapman C, Coulson K, Jeffery E, Preston S, et al: Development, implementation, and process evaluation of a regional palliative care quality improvement project. J Pain Symptom Manage 2009;38(4):483-495.

36. Gould DA, Lynn J, Halper D, Myers SK, Simon L, Holmes H: The New York City Palliative Care Quality Improvement Collaborative. Jt Comm J Qual Patient Saf 2007;33(6): 307-316.

37. Hanson LC, Schenck AP, Rokoske FS, Abernethy AP, Kutner JS, Spence C, Person JL: Hospices' preparation and practices for quality measurement. J: Pain Symptom Manage 2010;39(1):1-8.

38. Waller A, Girgis A, Currow D, Lecathelinais C: Development of the palliative care Needs Assessment Tool (PCNAT) for use by multi-disciplinary health professionals. Palliat Med 2008;22(8):956-964.

39. Remedios C, Thomas, K., Hudson, P: Psychosocial and bereavement support for family caregivers of palliative care patients: A review of the empirical literature. Melbourne: Centre for Palliaitve Care, 2011:71.

40. Council NHaMR: NHMRC levels of evidence and grades for recommendations for developers of guidelines. Canberra: National Health and Medical Research Council, 2009.

41. Murphy MK, Black NA, Lamping DL, McKee CM, Sanderson CF, Askham J, Marteau T: Consensus development methods, and their use in clinical guideline development. Health Technol Assess 1998;2(3):i-iv,1-88.

Address correspondence to: Peter Hudson, R.N., B.N. (Hons), Ph.D.

Centre for Palliative Care St. Vincent's Hospital P.O. Box 2900

Fitzroy VIC 3065

Australia

E-mail: phudson@unimelb.edu.au 